\title{
Guidelines for evaluation and
} management of cerebral collateral circulation in ischaemic stroke 2017

\section{Liping Liu, ${ }^{1}$ Jing Ding, ${ }^{2}$ Xinyi Leng, ${ }^{3}$ Yuehua $\mathrm{Pu},{ }^{1} \mathrm{Li}-$ An Huang, ${ }^{4}$ Anding $\mathrm{Xu},{ }^{4}$ Ka Sing Lawrence Wong, ${ }^{3}$ Xin Wang, ${ }^{2}$ Yongjun Wang, ${ }^{1}$ on behalf of the Chinese Society of Cerebral Blood Flow and Metabolism, the Chinese Stroke Association}

To cite: Liu L, Ding J, Leng X, et al. Guidelines for evaluation and management of cerebral collateral circulation in ischaemic stroke 2017. Stroke and Vascular Neurology 2018;3: e000135. doi:10.1136/svn2017-000135

Received 19 December 2017 Revised 10 April 2018

Accepted 12 April 2018 Published Online First 30 May 2018
Check for updates

${ }^{1}$ Department of Neurology, Beijing Tiantan Hospital, Capital Medical University, Beijing, China

2Department of Neurology, Zhongshan Hospital, Fudan University, Shanghai, China ${ }^{3}$ Department of Medicine and Therapeutics, The Chinese University of Hong Kong, Prince of Wales Hospital, Hong Kong, China

${ }^{4}$ Department of Neurology, The First Affiliated Hospital, Jinan University, Guangzhou, China

Correspondence to Professor Xin Wang; wang.xin@zs-hospital.sh.cn and Professor Yongjun Wang; yongjunwang1962@gmail.com

\section{ABSTRACT}

Collateral circulation plays a vital role in sustaining blood flow to the ischaemic areas in acute, subacute or chronic phases after an ischaemic stroke or transient ischaemic attack. Good collateral circulation has shown protective effects towards a favourable functional outcome and a lower risk of recurrence in stroke attributed to different aetiologies or undergoing medical or endovascular treatment. Over the past decade, the importance of collateral circulation has attracted more attention and is becoming a hot spot for research. However, the diversity in imaging methods and criteria to evaluate collateral circulation has hindered comparisons of findings from different cohorts and further studies in exploring the clinical relevance of collateral circulation and possible methods to enhance collateral flow. The statement is aimed to update currently available evidence and provide evidence-based recommendations regarding grading methods for collateral circulation, its significance in patients with stroke and methods under investigation to improve collateral flow.

\section{CONTEXT}

Good collateral circulation could enhance the benefit of endovascular treatment in acute ischaemic stroke and reduce the risk of relevant haemorrhagic transformation ${ }^{1-3}$; significantly reduce the risk of recurrent stroke in patients with symptomatic intracranial atherosclerotic stenosis (ICAS) ${ }^{4}$; and reduce the quantities and volume of infarction in ischaemic stroke. ${ }^{5}$ Accurate assessment of the structure and function of cerebral collateral circulation is an important prerequisite for individualised management of patients with stroke. Currently, assessment and intervention of collateral circulation in ischaemic stroke have been under active investigation. Various imaging criteria have been developed to gauge the collateral status and correlate with prognosis in patients with stroke. There are also emerging interventions to enhance collateral circulation in patients with stroke. Therefore, a writing group has been established under the Society of Cerebral Blood Flow and Metabolism, the Chinese Stroke
Association, for the current guideline on the evaluation and management of cerebral collateral circulation in ischaemic stroke. It is aimed to enhance general understanding of the cerebral collateral circulation among neurologists, neuroradiologists, neurointerventionalists and other relevant healthcare professionals, to provide evidence-based recommendations regarding collateral circulation in ischaemic stroke, and to promote future research in relevant areas. The current guideline is an update based on a previously published 'Chinese Consensus Statement on the Evaluation and Intervention of Collateral Circulation for Ischemic Stroke'.

\section{OVERVIEW}

Cerebral collateral circulation refers to the auxiliary vascular structures that compensate cerebral blood flow when 'normal' blood flow is impaired or restricted due to severe stenosis or occlusion of the principal supplying arteries or other focal or systemic situations. ${ }^{7}$ The status of collateral circulation is critical in determining the presence and volumes of penumbra and ischaemic core, which are important factors leading to heterogeneity in the time course and severity of individual ischaemic strokes. Recognition of the importance of collateral circulation and accurate assessment of the collateral status may facilitate better prognostication of patients with stroke and provide therapeutic implications.

Cerebral collateral circulation is usually divided into primary, secondary and tertiary collaterals. Primary collaterals refer to the arterial segments of the circle of Willis; secondary collaterals include the ophthalmic artery and leptomeningeal arteries, as well as other anastomoses between the distal, small-calibre arteries; and tertiary collaterals refer to newly developed microvessels through angiogenesis at the periphery of ischaemic regions. ${ }^{67}$ 
The concept of 'collaterome' has recently been proposed to represent 'the elaborate neurovascular architecture within the brain that regulates and determines the compensatory ability, response and outcome of cerebrovascular pathophysiology'. ${ }^{8}$ The concept involves the entire cerebral circulation system, including the arteries, veins and microvessels, and incorporates interactions between the cerebral vascular architecture, cerebral blood flow dynamics and tissue metabolism, and neuronal functions. ${ }^{8}$ It is a rising scientific field that urges cross-disciplinary efforts in relevant basic, translational and clinical research.

\section{IMAGING METHODS AND GRADING CRITERIA FOR CEREBRAL COLLATERAL CIRCULATION}

We herein summarise the imaging methods to assess the structure and function of cerebral collateral circulation.

Imaging methods to assess the structure of cerebral collateral circulation

Transcranial Doppler (TCD), transcranial colour-coded duplex sonography (TCCD), traditional single-phase CT angiography (CTA) or CTA-relevant methods such as CTA source image, CTA multiplanar reconstruction, CTA maximum intensity projection, timing-invariant CTA and multiphase CTA (or dynamic CTA), triphase CT perfusion (CTP), MR angiography (MRA) such as time-offlight MRA (TOF-MRA), phase-contrast MRA and quantitative MRA (QMRA), and digital subtraction angiography (DSA) have all been used in clinical practice and relevant research areas to assess the structure of cerebral collateral circulation. ${ }^{910}$ Among all these methods, DSA has been recognised as a gold standard to evaluate the collateral structure. However, due to the invasive nature and high cost of DSA, non-invasive imaging methods are more commonly used. Moreover, contrast injection during DSA exam may affect the blood flow rate and visibility of distal vessels, or even reverse the direction of blood flow within the circle of Willis, for example, the anterior or posterior communicating arteries.

TCD could non-invasively reflect real-time cerebral blood flow velocity, collateral status and cerebrovascular reactivity with a low cost, but the accuracy of TCD in diagnosing cerebrovascular abnormalities highly relies on the experience of the operators. ${ }^{11} 12$ Collateral flow through anterior communicating artery, posterior communicating artery, ophthalmic artery and leptomeningeal arteries could be directly or indirectly detected by TCD. The sensitivities of TCD in detecting a patent anterior communicating artery and collateral flow through basilar artery were reported to be $95 \%$ and $87 \%$, and the specificities were $100 \%$ and $95 \%$, respectively, with DSA as a reference standard. ${ }^{13}$ In addition, the flow diversion phenomenon in TCD, that is, high-velocity and low-resistance flow in the anterior cerebral artery (ACA) or posterior cerebral artery (PCA) in the presence of the middle cerebral artery (MCA) occlusion or severe stenosis, implies leptomeningeal collateral anastomoses between the ACA/PCA and the distal MCA branches. ${ }^{14}$ The sensitivity and specificity of flow diversion by TCD for predicting the presence of leptomeningeal collateral flow in DSA were, respectively, $81.1 \%$ and $76.7 \%$, and the positive and negative predictive values were, respectively, $70.8 \%$ and $85.2 \%$ in a previous report. ${ }^{14}$

TOF-MRA is another non-invasive method commonly used to assess the structure of cerebral collateral circulation. The reliability of TOF-MRA to assess leptomeningeal collaterals is limited by its relatively low spatial resolution. TOF-MRA is usually used to assess primary collaterals via the circle of Willis. In reference to DSA, the sensitivity and specificity of TOF-MRA in detecting collateral flow via the anterior part of the circle of Willis were $83 \%$ and $77 \%$, and $33 \%$ and $88 \%$ for the posterior part of the circle of Willis. ${ }^{15}$ A combination of TOF-MRA and TCD yielded a sensitivity of $92 \%$ and a specificity of $65 \%$ for detecting collateral flow via the anterior circle of Willis, and a sensitivity of $88 \%$ and a specificity of $41 \%$ for collateral flow via the posterior circle of Willis. ${ }^{15}$

CTA is also a non-invasive method that bears a high accuracy in assessing patency of the arterial segments in the circle of Willis, with $>90 \%$ agreement with DSA, but its sensitivity $(53 \%)$ is limited in depicting hypoplastic arterial segments. ${ }^{16}$ Blood flow via collaterals may delay as compared with normal antegrade flow. Thus, traditional single-phase CTA may underestimate compensating flow via collaterals. At present, timing-invariant $\mathrm{CTA}^{17}{ }^{18}$ and multiphase CTA (or dynamic CTA or four-dimensional CTA ${ }^{19-22}$ are increasingly used in clinical research to assess cerebral collateral status. Although such novel CTA methods could more accurately depict the collateral status and provide additional information such as the direction of the collateral flow, further investigation is needed before an extensive application in clinical practice.

\section{Imaging methods to assess the function of cerebral collateral} circulation

There are various imaging methods to evaluate the 'function' of cerebral collateral circulation, for instance, cerebrovascular reserve by TCD, xenon CT, single-photon emission CT (SPECT), positron emission tomography (PET), CTP, QMRA, traditional dynamic susceptibility contrast MR perfusion, arterial spin labelling (ASL), MR perfusion and others. These imaging methods usually gauge the cerebral blood flow direction/velocity/volume or perfusion status to reflect the blood flow compensating function of collaterals. Some novel imaging techniques could simultaneously reveal the structure and function of collateral circulation; for instance, QMRA could reveal directions of blood flow via collateral channels and quantify total/regional cerebral blood flow.

Rusanen et $a l^{23}$ used collateral circulation to predict infarct size and penumbra following thrombolytic therapy of acute ischaemic stroke. They used the Alberta Stroke Program Early CT Score (ASPECTS) of mean transit time (MTT) to evaluate the brain tissue at ischaemic 
risk and cerebral blood volume (CBV) score to evaluate the infarct core. The results showed that better MTT and ASPECTS score based on CBV correlated with better collateral circulation. A better collateral circulation is associated with a smaller infarct core and a larger mismatch ratio. ${ }^{24}$ CTP has been used to screen patients in the randomised controlled trial (RCT) for revascularisation. ${ }^{25-27}$ Some MR perfusion parameters have been used for the assessment of collateral status. The Endovascular Therapy Following Imaging Evaluation for Ischemic Stroke (DEFUSE 3) trial further added evidence on the benefit of perfusion imaging-based (CTP or MR perfusion mismatch) endovascular treatment in ischaemic stroke. ${ }^{27}$

\section{Commonly used grading scales for cerebral collateral circulation \\ DSA-based grading scales}

The most widely recognised grading system is the American Society of Interventional and Therapeutic Neuroradiology/Society of Interventional Radiology (ASITN/ SIR) collateral scale based on DSA, classifying the cerebral collateral status to grades $0-4$ as follows: grade 0 , no collaterals visible to the ischaemic site; grade 1 , slow collaterals to the periphery of the ischaemic site with persistence of some of the defect; grade 2, rapid collaterals to the periphery of ischaemic site with persistence of some of the defect and to only a portion of the ischaemic territory; grade 3, collaterals with slow but complete angiographic blood flow of the ischaemic bed by the late venous phase; and grade 4, collaterals with slow but complete angiographic blood flow of the ischaemic bed by the late venous phase. ${ }^{28}$ Grades $0-1,2$ and $3-4$ are usually regarded as poor, moderate and good collateral flow. The ASITN/SIR collateral grading system has been demonstrated to be reliable in assessing the collateral status in patients with stroke in a number of multicentre studies.

The Endovascular Stroke Treatment (ENDOSTROKE) registry was an international, multicentre study recruiting adult patients with acute ischaemic stroke and intracranial large artery occlusion for whom mechanical revascularisation procedure was attempted. ${ }^{29}$ Among the 160 patients with acute proximal MCA occlusion in the ENDOSTROKE registry, the ASITN/SIR collateral scale was used to gauge the collateral status to correlate with the imaging and clinical outcomes after acute endovascular treatment. The investigators found a positive correlation between a better collateral status and a higher reperfusion rate, leading to a smaller infarct volume and a better clinical outcome. The rates of achieving successful reperfusion by the Thrombolysis in Cerebral Infarction Scale $2 b$ or 3 among those with ASITN/SIR collateral grades of 0-1, 2 or 3-4 were, respectively, $21 \%, 48 \%$ and $77 \%(\mathrm{p}<0.001)$. The proportion of the infarcts smaller than one-third of the MCA territory (32\%, $48 \%$ and $69 \%$ for collateral grades $0-1,2$ or $3-4 ; p<0.001)$, and more importantly the proportion of patients with a good functional outcome at least 3 months after the intervention $(11 \%, 35 \%$ and $49 \%$ for collateral grades $0-1,2$ or $3-4 ; \mathrm{p}=0.007$ ), were both significantly higher in those with better collaterals. Multivariate analysis reinforced the role of collateral status as an independent predictor for reperfusion, infarct size and long-term functional outcomes in patients receiving endovascular treatment for acute proximal MCA occlusion. ${ }^{29}$ Another subgroup analysis of the ENDOSTROKE registry of 148 patients with acute basilar artery occlusion also indicated the predictive value of collateral status by the ASITN/SIR collateral scale for reperfusion and clinical outcomes. ${ }^{30}$ In addition, post-hoc analysis of the Interventional Management of Stroke III (IMS III) ${ }^{31}$ and Solitaire FR With the Intention for Thrombectomy $^{32}$ trials' data showed similar results.

Christoforidis et $a l^{33}$ proposed another collateral grading system based on DSA that is less frequently used, which classifies the collateral status to five grades: grade 1 , collaterals reconstituted the entire distal portion of the occluded vessel segment; grade 2, collaterals reconstituted vessels in the proximal portion of the segment adjacent to the occluded vessel; grade 3, collaterals reconstituted vessels in the distal portion of the segment adjacent to the occluded vessel; grade 4, collaterals reconstituted vessels two segments distal to the occluded vessel; and grade 5, little or no significant reconstitution of the territory of the occluded vessel. Good collateral status by this grading system (grades 1 or 2) has been correlated with smaller infarct volume, lower risk of haemorrhagic transformation and lower modified Rankin Scale (mRS) at discharge, in patients with ischaemic stroke receiving intra-arterial thrombolysis in relatively small-scale studies. ${ }^{33} 34$ This collateral grading method is not commonly used in clinical practice.

\section{CTA-based grading scales}

The Endovascular Treatment for Small Core and Proximal Occlusion Ischemic Stroke (ESCAPE) study ${ }^{2}$ is an international multicentre RCT study evaluating thrombectomy, with a primary prognostic indicator of functional outcome at 90 days after onset. The results showed that for patients with acute ischaemic stroke with favourable collateral circulation, based on multiphase CTA ASPECTS collateral circulation score (5-4 points), prompt administration of endovascular treatment improved functional outcome (mRS score at 90 days) and reduced mortality.

Table 1 shows examples of other collateral grading methods based on CTA, including the methods proposed by Miteff $e t a l^{35}$ (grading collateral flow distal to MCA occlusion), Maas et $a l^{36}$ (assessing collaterals at the Sylvian sulcus and cerebral convexity, as well as collateral pathways via the circle of Willis), Tan et $a l^{37}$ (grading collaterals in the MCA territory), the regional leptomeningeal collateral (rLMC) score $^{38}$ (assessing collaterals in MCA cortical regions, parasagittal ACA territory, the basal ganglia and the Sylvian sulcus), and the ACA-MCA and PCA-MCA regional collateral score. ${ }^{39} 40$ There are also modified versions of originally DSA-based collateral 
Table 1 Examples of collateral grading methods based on CTA

\section{Collateral \\ grading \\ methods}

Miteff collateral Collateral status is graded in maximum intensity projection reconstructions of single-phase CTA in axial,

grading method ${ }^{35}$ coronal and sagittal planes in patients with MCA occlusion, and graded as:

- Good, if major MCA branches are reconstituted distal to the occlusion.

- Moderate, if some MCA branches are shown in the Sylvian fissure.

- Poor, if only the distal superficial MCA branches are reconstituted.

Maas collateral Collateral vessels in the Sylvian fissure and the leptomeningeal

grading method ${ }^{36}$ convexity are graded in CTA source images by comparing the

symptomatic hemisphere with the contralateral unaffected hemisphere as:

$1=$ Absent.

$2=$ Less than the contralateral normal side.

$3=$ Equal to the contralateral normal side.

$4=$ Greater than the contralateral normal side.

$5=$ Exuberant.
The presence and status of the anterior and posterior communicating arteries are graded as:

$1=$ Absent.

2=Probably present.

$3=$ Hairline.

$4=$ Definitely present.

$5=$ Robust.

\begin{abstract}
Tan collateral
Leptomeningeal collateral status is graded in source images and maximum intensity projection

grading system ${ }^{37}$

$0=$ No collateral supply to the occluded MCA territory.

$1=$ Collateral supply filling $\leq 50 \%$ of the occluded MCA territory.

$2=$ Collateral supply filling $>50 \%$ but $<100 \%$ of the occluded MCA territory.

$3=100 \%$ collateral supply of the occluded MCA territory.

Regional

leptomeningeal

collateral (rLMC) score $^{38}$

The rLMC score (20 points) compares the extent of contrast opacification in arteries distal to an $\mathrm{M} 1$

MCA occlusion ( \pm internal carotid artery occlusion) in the symptomatic hemisphere with the contralateral

hemisphere in multiplanar reformatted CTA, with a higher score indicating a better collateral status.

The extent of contrast opacification is scored as 0 (artery not seen), 1 (less prominent) or 2 (equal or more

prominent than the opposite hemisphere) for the six ASPECTS cortical regions (M1-6), parasagittal ACA

territory and the basal ganglia, while pials in the Sylvian sulcus are given a higher score, that is, 0, 2 or 4.

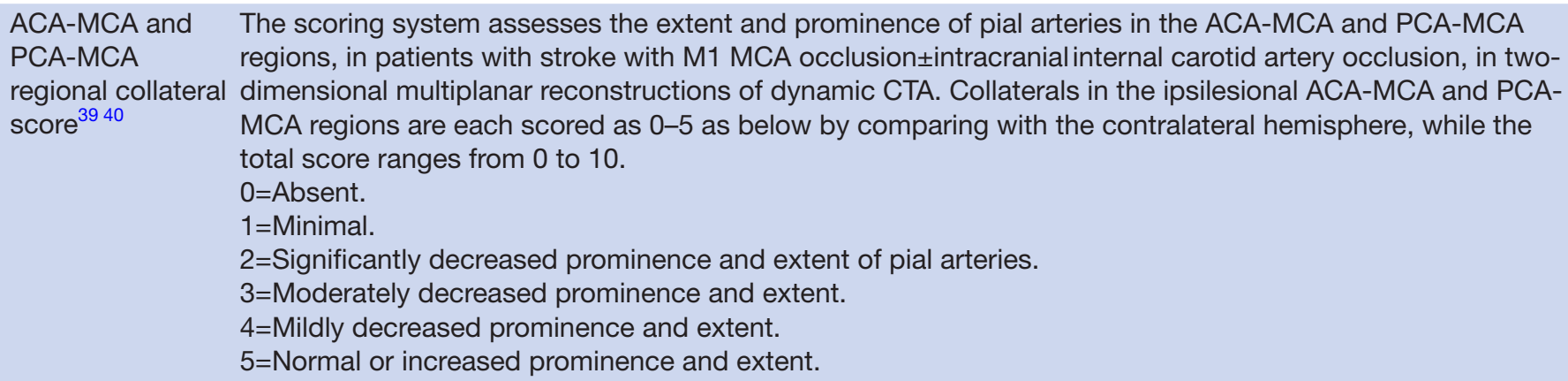

ACA, anterior cerebral artery; ASPECTS, the Alberta Stroke Programme Early CT Score; CTA, CT angiography; MCA, middle cerebral artery; PCA, posterior cerebral artery.

grading methods for CTA, such as the ASITN/SIR collateral scale ${ }^{28}$ for CTA and the Christoforidis collateral grading system $^{33}$ for CTA, which are not listed in table 1 . There have been studies comparing the clinical relevance of these grading methods, but the findings were heterogeneous and none of the collateral grading systems have been well validated in large-scale studies. Further investigation is needed to establish an optimal method to non-invasively assess collateral circulation in patients with stroke.

The predictive values of the ACA-MCA and PCA-MCA regional collateral score,${ }^{3940}$ and the Maas $e t a l^{36}$ and Tan $e t$ $a l^{37}$ collateral grading methods for a favourable 3-month functional outcome after intravenous thrombolysis and/ or endovascular treatment, among patients with acute stroke with M1 MCA occlusion \pm intracranial internal carotid artery (ICA) occlusion, were tested in 185 patients from the IMS III cohort. In multivariate analyses, collateral status by each of the collateral scales was significantly, independently correlated with an mRS of $0-2$ at 3 months after treatment. ${ }^{40}$

Another study compared the abilities of the Miteff $e t$ $a l l^{35}$ Maas $e t a l^{36}$ and Tan $e t a l^{37}$ collateral grading methods and the rLMC score to predict the 3-month functional outcomes in acute anterior circulation stroke treated with intravenous thrombolysis. Among 200 patients, only good collateral status by the Miteff collateral grading method was found to be an independent predictor for 
a favourable functional outcome (mRS 0-1) at 3 months (OR, 3.34; 95\% CI 1.24 to 9.00; $\mathrm{p}=0.01$ ). In addition, poor collateral status by the Miteff method, the Maas method and the rLMC score were all independently related to an extremely poor functional outcome (mRS 5-6) at 3 months. ${ }^{41}$

A more recent study has compared four different CTA-based collateral scales in predicting the volume of infarct core and the perfusion:diffusion mismatch ratio within the first few hours after an ischaemic stroke among 30 patients with acute M1 MCA or terminal carotid artery occlusion. The ACA-MCA and PCA-MCA regional collateral score ${ }^{3940}$ and a modified version of the ASITN/SIR collateral scale ${ }^{28}$ for dynamic CTA both showed good correlations with early infarct core volume (Spearman's correlation coefficients both around $-0.7 ; \mathrm{p}<0.001$ ) and the mismatch ratio (Spearman's correlation coefficients both around 0.6; $\mathrm{p}<0.001)$. However, the Miteff collateral grades, or a modified version of the Christoforidis collateral grading system ${ }^{33}$ for dynamic CTA, were not significantly linearly correlated with the infarct core volume and the mismatch ratio. ${ }^{42}$

\section{Recommendations}

1. Different imaging modalities could be used to evaluate the cerebral collateral status in patients with ischaemic stroke or transient ischaemic attack (TIA). By far, DSA is a gold standard in the assessment of the presence and extent of primary and secondary collaterals. In non-invasive imaging modalities to assess the presence and extent of secondary collateral circulation, CTA is more reliable than MRA (class II; level of evidence C).

2. For patients with acute ischaemic stroke eligible for endovascular treatment, evaluation of the cerebral collateral circulation status by the ASITN/SIR collateral scale in DSA is reasonable, which helps predict the risk and benefit of acute endovascular treatment (class I; level of evidence A); multiphase CTA or perfusion imaging could also be used to assess the cerebral collateral circulation prior to endovascular treatment in such patients (class I; level of evidence B).

3. There is no general agreement regarding an optimal collateral grading system in ischaemic stroke based on non-invasive imaging modalities. The reliability and the clinical significance, such as the predictive values for prognosis of ischaemic stroke, of the currently available grading systems need further investigation.

\section{COLLATERAL CIRCULATION AND PROGNOSIS OF ISCHAEMIC STROKE}

Collateral circulation and hyperacute reperfusion therapies in stroke

Hyperacute reperfusion therapies for ischaemic stroke include intravenous thrombolysis and endovascular therapies, and intravenous intra-arterial bridging therapies, while endovascular therapies usually refer to intra-arterial thrombolysis and mechanical thrombectomy. As a mainstay of early treatment in acute ischaemic stroke, timely restoration of cerebral blood flow salvages the ischaemic penumbra, improves functional outcome and reduces mortality, ${ }^{4344}$ and is recommended in the American and Chinese guidelines as the first-line treatment for eligible patients presenting within corresponding time windows. ${ }^{43-46}$ The status of cerebral collateral circulation has significant predictive values for the imaging and clinical outcomes of patients with stroke receiving such treatment.

\section{Collateral circulation and intravenous thrombolysis in stroke}

Intravenous thrombolysis is the first-line treatment for patients with acute ischaemic stroke presenting within 4.5 hours without contraindications. ${ }^{43}$ By far, there have been few prospective studies investigating the role of collateral circulation in determining outcomes of patients receiving intravenous thrombolytic therapy. Yet post-hoc analysis of several RCTs indicated that a better collateral status prior to intravenous thrombolysis was associated with less severe clinical symptoms (the Combined Lysis of Thrombus in Brain Ischemia Using transcranial Ultrasound and Systemic TPA trial, CLOTBUST),${ }^{47}$ a smaller infarct core in diffusion-weighted MRI and a larger diffusion-perfusion mismatch (Echoplanar Imaging Thrombolytic Evaluation Trial). ${ }^{48}$ More importantly, better collaterals at baseline were associated with a higher incidence of achieving a favourable functional outcome at 3 months after the treatment (CLOTBUST and IMS III trials). ${ }^{40} 47$

A recent systematic review and meta-analysis synthesised evidence regarding the impact of pretreatment collateral circulation on the outcomes of patients with stroke treated with intravenous thrombolysis. ${ }^{49}$ Overall, 28 primary studies of 3057 patients were included in the analysis, including 25 cohort studies (mostly retrospective) and 3 post-hoc analyses of RCTs. Meta-analysis based on these data has demonstrated a favourable role of better collateral circulation in this subset of patients, including a lower risk of symptomatic intracranial haemorrhage (risk ratio (RR) $0.38 ; 95 \%$ CI 0.16 to $0.90 ; \mathrm{p}=0.03$ ), a higher incidence of early neurological improvement (RR $4.21 ; 95 \%$ CI 1.57 to $11.28 ; \mathrm{p}=0.004$ ) and a higher frequency of a favourable functional outcome (mRS $0-2$ or $0-1$ as defined in different primary studies) at 3-6 months after the thrombolytic treatment (RR 2.45; $95 \%$ CI 1.94 to $3.09 ; \mathrm{p}<0.001) .{ }^{49}$ Such findings may be attributed to a lower National Institutes of Health Stroke Scale score (NIHSS; mean difference 6.6; 95\% CI 4.4 to $8.7 ; \mathrm{p}<0.001)$ and a smaller infarct volume in patients with better collateral circulation. However, no significant correlation was identified between the baseline collateral status and successful reperfusion or recanalisation after intravenous thrombolytic therapy (RR 1.34; 95\% CI 0.87 to 2.07; $\mathrm{p}=0.19$ ). Unfortunately, data are limited to allow quantitative synthesis of the correlations between baseline collateral status and the overall risk of haemorrhagic transformation (symptomatic or asymptomatic), the final infarct volume and death risk at 3 months after treatment. ${ }^{49}$ 
In summary, previous studies show that pretreatment collateral status plays an important role in determining short-term and long-term outcomes of patients with stroke receiving intravenous thrombolytic therapy, while further prospective investigations are needed before more confirmative conclusions could be drawn.

\section{Collateral circulation and endovascular treatment in stroke}

A number of previous trials failed to prove the superiority of endovascular treatment over routine medical treatment with or without intravenous thrombolysis in acute ischaemic stroke. In 2015, several pivotal RCTs demonstrated the safety and efficacy of endovascular treatment in ischaemic stroke with cervical or intracranial arterial occlusion, when the American and Chinese guidelines on early management of ischaemic stroke were updated, recommending endovascular treatment for eligible patients presenting within 6 hours of symptom onset with or without prior intravenous thrombolytic therapy. ${ }^{44}{ }^{46}$ Except for the application of newer generation thrombectomy devices, adding imaging eligibility criteria for patient selection, for example, a moderate-to-good collateral circulation, a smaller infarct core or evidence of salvageable brain tissue, may have contributed to the positive findings in these more recent RCTs. ${ }^{2} 25$ 50-53 In 2017, encouraging evidence has emerged that patients with ischaemic stroke with cervicocerebral artery occlusion may benefit from endovascular treatment up to 24 hours after stroke onset. For instance, the Diffusion-Weighted Imaging or Computerized Tomography Perfusion Assessment with Clinical Mismatch in the Triage of Wake Up and Late Presenting Strokes Undergoing Neurointervention (DAWN) trial enrolled patients with occlusion of the ICA and/or the first segment of the MCA in 6-24hours, and had a mismatch between the severity of the clinical deficit and the infarct volume, which was assessed with the use of diffusion-weighted MRI or perfusion CT. The DAWN trial witnessed a significant increase (an absolute increase of 35\%) in the incidence of a 90-day favourable functional outcome (mRS 0-2) in patients with intracranial ICA or M1 MCA occlusion, yet a small infarct core treated with mechanical thrombectomy 6-24hours after the stroke onset as compared with routine medical treatment. ${ }^{52} 53$ Moreover, the CT Perfusion to Predict Response to Recanalization in Ischemic Stroke trial revealed that baseline CTP 'target mismatch', which had a small ischaemic core and a large penumbra, indicated a significant improvement in the NIHSS score even in those treated up to 18 hours after symptom onset. ${ }^{26}$ The DEFUSE 3 trial indicates that endovascular thrombectomy for patients with ischaemic stroke 6-16hours with proximal MCA or internal carotid artery occlusion, an initial infarct size of less than $70 \mathrm{~mL}$, and a ratio of the volume of ischaemic tissue on perfusion imaging to infarct volume of 1.8 or more had a significant favourable functional outcomes on the mRS at 90 days compared with the medical therapy-alone group (OR, 2.77; $<<0.001){ }^{27}$

To enlarge the benefit of endovascular treatment within or beyond the 6-hour time window as recommended by current guidelines, it is also essential to find the 'right' patients to treat, while good pretreatment collaterals may play an important role in preserving salvageable tissue. Two recent systematic reviews and meta-analyses investigated the effects of pretreatment collateral circulation in governing clinical and imaging outcomes of patients with stroke receiving endovascular treatment. ${ }^{545}$ Based on data from over 20 studies of $>2000$ patients with stroke treated with intra-arterial thrombolysis and/or mechanical thrombectomy, with or without prior intravenous thrombolysis, better pretreatment collateral circulation is associated with slightly higher rates of successful recanalisation (RR 1.23 ; $95 \%$ CI 1.06 to $1.42 ; \mathrm{p}=0.006$ ) and reperfusion (RR $1.28 ; 95 \%$ CI 1.17 to $1.40 ; \mathrm{p}<0.001),{ }^{55}$ a significantly lower risk of symptomatic intracranial haemorrhage within 7 days or before discharge (RR 0.59; $95 \%$ CI 0.43 to $0.81 ; \mathrm{p}=0.001$ ), a doubled chance of achieving a favourable functional outcome at 3 months (RR 1.98; 95\% CI 1.64 to 2.38; $\mathrm{p}<0.001$ ), and a halved risk of death at 3 months (RR $0.49 ; 95 \%$ CI 0.38 to $0.63 ; \mathrm{p}<0.001) .{ }^{54}$ Although the mechanisms underlying the protective effects of collateral circulation in such patients have not been well illustrated, inferences are that collateral circulation via the circle of Willis or pial arteries compensates cerebral blood flow adjacent to the ischaemic area, which provides better access of the clot to intrinsic and extrinsic thrombolytic agents and possibly a back pressure that facilitates dislodgement of the clot. It may also mitigate the ischaemia-reperfusion injuries. ${ }^{5657}$

\section{Collateral circulation and symptomatic ICAS}

ICAS is of high prevalence in the Chinese population, which is a major cause of ischaemic stroke and TIA in China and other Asian countries. ${ }^{589}$ For instance, in the Chinese Intracranial Atherosclerosis (CICAS) study, $46.6 \%$ of the 2864 patients with ischaemic stroke or TIA had ICAS.$^{60}$ In the 1089 patients with MRA images in the Clopidogrel in High-Risk Patients with Acute Nondisabling Cerebrovascular Events trial, 608 (55.8\%) had ICAS. ${ }^{61}{ }^{62}$ According to post-hoc analysis of the Warfarin-Aspirin Symptomatic Intracranial Disease (WASID) trial, the collateral status significantly altered the risk of recurrent stroke in patients with symptomatic ICAS. ${ }^{4}$ To further verify the impact of collateral circulation on the recurrent risk and functional outcomes of patients with symptomatic ICAS, we systematically searched PubMed for full-text publications between 1 January 2000 and 9 September 2017, and retrieved 457 relevant records. Seven of these publications reported correlations between the collateral status and prognosis of patients with ICAS, ${ }^{60} 63-68$ including post-hoc analyses of the CICAS $^{60}$ and WASID data. ${ }^{63}$ 
Collaterals via the circle of Willis and outcomes of patients with symptomatic ICAS

There is no confirmative conclusion regarding the effect of collaterals via the circle of Willis on the risk of recurrence and the functional outcomes of patients with symptomatic ICAS. The largest study by far reporting the correlation between the completeness of the circle of Willis and the recurrence risk in patients with ischaemic stroke or TIA was CICAS. ${ }^{60}$ Patients with stroke or TIA with a complete circle of Willis had a higher risk of recurrence within 1 year, as compared with those without (adjusted HR 2.36; 95\% CI 1.19 to $4.69 ; \mathrm{p}=0.015$ ). However, such findings were generated from an overall analysis of CICAS, including 1335 patients with and 1529 patients without ICAS. Thus, no conclusion could be drawn from such analysis concerning the protective or harmful effect of collateral flow through the circle of Willis in patients with ICAS. ${ }^{60}$ There has been another small-scale study reporting that a complete circle of Willis reduced the risk of recurrence within 3 years among patients with 70\%-99\% symptomatic ICAS who received medical treatment, which did not affect the recurrent risk among 43 patients who received angioplasty with/without stenting therapy. ${ }^{69}$

\section{Leptomeningeal and other collateral pathways and outcomes of patients with symptomatic ICAS}

Among 569 patients recruited to the WASID trial with $50 \%-99 \%$ symptomatic atherosclerotic stenosis of a major intracranial artery, who were treated with antiplatelet or anticoagulant therapies, adequate angiographic data to assess the leptomeningeal collaterals were available in 287 patients. The angiogram-based collateral extent independently predicted recurrent ischaemic stroke in the symptomatic arterial territory (HR for none vs good collaterals, 1.14; $95 \%$ CI 0.39 to 3.30; HR for poor vs good collaterals, 4.36; $95 \%$ CI 1.46 to $13.07 ; \mathrm{p}<0.0001)$. Subgroup analyses by the severity of arterial stenosis indicated that more robust leptomeningeal collaterals were associated with a lower risk of recurrence among patients with 70\%-99\% symptomatic ICAS (HR none vs good, 4.60; 95\% CI 1.03 to 20.56; HR poor vs good, $5.90 ; 95 \%$ CI 1.25 to 27.81; $\mathrm{p}=0.0427$ ), which, however, were associated with an increased risk of recurrence in those with 50\%-69\% symptomatic ICAS (HR none vs good, 0.18 ; $95 \%$ CI 0.04 to 0.82 ; HR poor vs good, 1.78 ; $95 \%$ CI 0.37 to $8.57 ; \mathrm{p}<0.0001){ }^{4}$

A small-scale, single-centre study of 69 patients with 50\%-100\% symptomatic ICAS found that those with better leptomeningeal collateral compensations (ASITN/ SIR collateral flow grades 2-4 vs $0-1$ ) had a better chance to achieve a favourable functional outcome at 3 months (adjusted OR 7.50; 95\% CI 1.11 to 50.7; $\mathrm{p}=0.04$ ) and a lower risk of recurrent ischaemic stroke or TIA within 1 year (OR $0.18 ; 95 \%$ CI 0.04 to $0.96 ; \mathrm{p}=0.04) .{ }^{64}$

Another small study of 88 patients with symptomatic MCA occlusion implied that better collaterals as defined by the presence of hyperintensities in the Sylvian fissure on fluid-attenuated inversion recovery sequence were independently correlated with a lower risk of poor functional outcome (mRS 3-6) at 3 months (adjusted OR $0.272 ; 95 \%$ CI 0.101 to $0.733 ; \mathrm{p}=0.010$ ). However, MCA occlusions in this study were attributed to various aetiologies, among which only $40 \%$ were of atherosclerotic origin. $^{66}$

Lee $e t a l^{68}$ modified the ASITN/SIR collateral flow grades to assess the extent of vessel filling in the superior cerebellar artery territory (scores $0-4$ ) and the anterior/posterior inferior cerebellar artery territory (scores $0-4)$, with a total score of $0-8$, among 98 patients with symptomatic, atherosclerotic basilar artery stenosis (70\%-99\%). Better collateral status by such method was associated with a reduced incidence of poor functional outcome (mRS 3-6) at 3 months (OR of 1-score increment in the collateral score, $0.21 ; 95 \%$ CI 0.08 to 0.58 ; $\mathrm{p}=0.003){ }^{68}$

Except for the collateral pathways as mentioned above, the effect of the presence of the anterior temporal artery in patients with symptomatic MCA occlusion was investigated in 98 patients, which was significantly correlated with a favourable functional outcome (mRS 0-2) at 3 months (adjusted OR 4.45; 95\% CI 1.52 to 13.03; $\mathrm{p}=0.007$ ), independent of the baseline NIHSS score, the infarct size and pattern. ${ }^{65}$

\section{Recommendations}

1. For patients with acute ischaemic stroke with cervicocerebral arterial occlusion who receive intravenous, intra-arterial or intravenous intra-arterial bridging reperfusion therapies, the pretreatment cerebral collateral status possesses significant prognostic values for the outcomes (class I; level of evidence B).

2. Based on current evidence, assessment of the collateral status and infarct core helps identify patients who will benefit from such treatment, especially among those presenting beyond 6 hours after symptom onset (class I; level of evidence B).

3. For patients with symptomatic ICAS, the collateral status predicts the risk of recurrent stroke and the functional outcome (class I; level of evidence B).

4. The leptomeningeal collateral status could significantly alter the risk of recurrent stroke and the functional outcome of patients with symptomatic ICAS, but its possibly diverging effects in patients with different degrees of stenosis need to be validated in further studies (class IIb; level of evidence B).

5. There is no confirmative conclusion regarding the effect of collaterals via the circle of Willis on the risk of recurrence and the functional outcomes of patients with symptomatic ICAS, which warrants further investigation (class IIb; level of evidence B).

6. Prospective, registry studies based on non-invasive imaging methods to assess the collateral circulation may further reveal the role of collateral circulation in patients with acute ischaemic stroke who opted for hyperacute reperfusion therapies, or those with symptomatic ICAS or ischaemic stroke of other subtypes (class I; level of evidence $\mathrm{C}$ ). 
INTERVENTIONS TO ENHANCE CEREBRAL COLLATERAL CIRCULATION IN ISCHAEMIC STROKE

Non-pharmacological interventions

Extracranial-intracranial bypass surgery

Extracranial-intracranial (EC-IC) bypass surgery may improve haemodynamic parameters in patients with symptomatic cervicocerebral artery stenosis or occlusion. ${ }^{70} \mathrm{~A}$ large RCT (1377 patients) conducted over 30 years ago, the EC/IC Bypass Study, indicated the inferiority of direct EC-IC bypass surgery over medical treatment among patients with steno-occlusive disease of the extracranial and/or intracranial arteries. ${ }^{71}$ From 2002 to 2010, the Carotid Occlusion Surgery Study (COSS) trial compared direct EC-IC bypass surgery plus medical treatment versus medical treatment alone among patients with symptomatic atherosclerotic internal carotid artery occlusion, who had haemodynamic cerebral ischaemia as defined by an increased ipsilateral:contralateral oxygen extraction fraction ratio on PET. ${ }^{72}$ The COSS trial was prematurely terminated due to futility-the rates of stroke or death within 30 days and ipsilateral ischaemic stroke within 2 years were not significantly different between the surgical and non-surgical groups (21.0\% vs $22.7 \%$; $=0.78) .{ }^{72}$ The Japanese EC-IC Bypass Trial (JET), recruiting patients between 1998 and 2002, had a similar study design with that of COSS, but the JET trial defined the cerebral haemodynamic compromise by decreased cerebral blood flow and deceased cerebrovascular reactivity to vasodilation in PET or SPECT. ${ }^{73}$ The JET trial reported a lower rate of the primary endpoint in the surgical than in the non-surgical groups among 196 patients $(\mathrm{p}=0.046)$. However, there have been concerns over the results of JET, since there was zero primary endpoint in the surgical group within the first month after the EC-IC bypass surgery in JET, which was $15 \%$ in the surgical group of the COSS trial. $^{72} 73$ Overall, based on currently available evidence, direct EC-IC bypass surgery is not recommended for patients with ischaemic stroke or TIA and ipsilateral, atherosclerotic ICA or MCA stenosis or occlusion in the 2014 American Heart Association/American Stroke Association guidelines for secondary stroke prevention. ${ }^{74}$

Encephaloduroarteriosynangiosis (EDAS), an indirect EC-IC bypass surgical method, has recently been reported safe and possibly effective in improving collateral circulation and reducing risk of recurrence, among small groups of patients with symptomatic ICAS with refractory stroke despite the best medical treatment. ${ }^{75} 76$ A prospective, single-arm clinical trial, the EDAS (Surgical) Revascularization for Symptomatic Intracranial Arterial Stenosis trial (ClinicalTrials.gov identifier: NCT01819597), is investigating the safety and efficacy of EDAS in these patients.

\section{Partial aortic occlusion by the NeuroFlo technology}

The NeuroFlo Catheter has two balloons which when mounted and inflated in the aorta could partially occlude the aortic lumen above and below the renal arteries, to increase cerebral blood flow. The Safety and Efficacy of NeuroFlo in Acute Ischemic Stroke (SENTIS) trial is the largest trial comparing the NeuroFlo technique with standard medical treatment among patients with acute cortical ischaemic stroke. ${ }^{77}$ It demonstrated in 515 patients that partial aortic occlusion by NeuroFlo Catheter to increase cerebral blood flow is safe among patients with stroke ( $p$ value for comparison of serious adverse events between the two groups $=0.923$ ). There was no significant difference between the two groups in the primary efficacy outcome, a favourable function outcome (OR 1.17; $95 \%$ CI 0.81 to $1.67 ; \mathrm{p}=0.407$ ), but there was a trend of decreased all-cause mortality in the NeuroFlo-treated group (11.2\% vs $16.3 \%$; OR 1.60 ; $95 \%$ CI 0.91 to 2.83 ; $\mathrm{p}=0.086) .{ }^{77}$ Subsequent subgroup analysis of the SENTIS data showed that patients aged over 70 years, patients who were treated with NeuroFlo within 5 hours of stroke onset and patients with moderate stroke severity (NIHSS scores of 8-14) may benefit more from the NeuroFlo treatment than medical treatment. ${ }^{77} 78$ Selecting appropriate patients is important for the NeuroFlo treatment to benefit, while relevant findings need further verification.

\section{External counterpulsation}

External counterpulsation (ECP) is a non-invasive method that enhances cardiac output and blood flow to vital organs including the brain, by inflating pressure cuffs around the lower extremities and the buttocks during the diastole and deflating the cuffs during the systole. ECP treatment is safe and feasible in patients with ischaemic stroke. ${ }^{79-81}$ It could augment cerebral blood flow in the ipsilateral and contralateral hemispheres among patients with stroke with large artery occlusive disease, which may imply enhanced collateral flow to the ischaemic territories. ${ }^{82}$ A single session of ECP (1 hour) may be associated with transient improvement in the neurological symptoms of patients with stroke, according to the Counterpulsation to Upgrade Forward Flow in Stroke trial (23 patients). ${ }^{81}$ Another pilot study of 50 patients with ischaemic stroke with large artery occlusive disease showed a slightly more significant decrease in the NIHSS score (2.1 vs 1.3; $\mathrm{p}=0.061$ ) after 35 daily sessions of ECP treatment (1 hour per session) than no ECP treatment. ${ }^{80}$ Therefore, ECP is a safe and possibly effective method to enhance cerebral blood flow and improve outcomes of patients with stroke, which warrants further investigation.

\section{Lying-flat head positioning}

Cerebral autoregulation may be impaired in patients with ischaemic stroke, especially in the affected cerebral hemisphere. Thus, a lying-flat head positioning may increase cerebral blood flow through collateral circulation or gravity, as compared with an upright head positioning. ${ }^{83}$ A systematic review and meta-analysis of four small studies (57 patients in total) indicated that ipsilesional but not contralesional MCA flow velocities were significantly higher when patients were in a lying-flat head position at $0^{\circ}$ or $15^{\circ}$ as compared with an upright head position of $30^{\circ} .{ }^{84}$ The mean flow velocity of ipsilesional MCA increased by $8.3 \mathrm{~cm} / \mathrm{s}$ on average with a head position 
from $30^{\circ}$ to $0^{\circ}(95 \%$ CI 5.3 to $11.3 \mathrm{~cm} / \mathrm{s} ; \mathrm{p}<0.001)$ and $4.6 \mathrm{~cm} / \mathrm{s}$ from $30^{\circ}$ to $15^{\circ}$ (95\% CI 2.9 to $6.2 \mathrm{~cm} / \mathrm{s}$; $\mathrm{p}<0.001){ }^{84}$ The Head Position in Acute Stroke Trial (HeadPoST) investigated the effects of different head positions in altering outcomes of over 11000 patients with acute ischaemic or haemorrhagic stroke who were nursed to a lying-flat or sitting-up $\left(\geq 30^{\circ}\right)$ head positions and remaining in the position for 24 hours. ${ }^{85}$ Unfortunately, the HeadPoST study did not show any difference in the lying-flat and sitting-up head positions in affecting the 3-month function outcome, in the overall analysis or in subgroup analyses according to stroke subtypes, initial stroke severity, age and others. ${ }^{86}$ Of note, HeadPoST did not assess arterial occlusion status in patients with stroke, which reduced power to detect a benefit of lying flat. Therefore, no conclusion could be drawn based on current evidence regarding the effects of different head positions on clinical outcomes of patients with ischaemic stroke.

\section{Other non-pharmacological interventions}

Remote limb ischaemic preconditioning (RIPC) may condition remote vital organs including the brain for subsequent ischaemic events, by inducing transient episodes of mild ischaemia in the limbs. There have been preliminary studies indicating that long-term, repeated RIPC of bilateral arms is safe and feasible in patients with stroke aged under or above 80 years who had symptomatic ICAS. Compared with standard medical treatment alone, RIPC plus standard medical treatment may reduce the risk of recurrent stroke or TIA in such patients by improving cerebral perfusion and relieving the inflammation stress. ${ }^{87} 88$ Large prospective studies are needed to further explore the efficacy of RIPC in patients with stroke, for instance, the Remote Ischemic Conditioning for Avoiding Recurrence of Ischemic Stroke in Patients with Symptomatic Intracranial Atherosclerotic Stenosis trial (ClinicalTrials.gov identifier: NCT02534545), which is currently under way.

In addition, there are novel methods that have shown promising effects in enhancing cerebral collateral circulation and cerebral blood flow in experimental stroke models, such as inhaling nitric oxide, ${ }^{89}$ stimulating the sphenopalatine ganglion ${ }^{90}$ and others. But more evidence is needed before testing these methods in patients with stroke.

\section{Pharmacological interventions \\ Statins}

Statin therapy has been demonstrated to have a protective effect in preventing stroke in patients with stroke, TIA or coronary artery disease. The relative risk reduction of statins versus placebo for a stroke event during follow-up in previous RCTs ranged from below 5\% to over $30 \% .{ }^{91}$ A recent systematic review and meta-analysis has demonstrated that prestroke statin use is associated with milder initial stroke severity (OR 1.24; 95\% CI 1.05 to 1.48; $\mathrm{p}=0.013$ ), better functional outcome (OR 1.50;
$95 \%$ CI 1.29 to $1.75 ; \mathrm{p}<0.001)$ and lower mortality (OR $0.42 ; 95 \%$ CI 0.21 to $0.82 ; \mathrm{p}=0.011)$. In-hospital statin use is also associated with better functional outcome and lower mortality. Among patients with stroke treated with thrombolytic therapy, statin use also leads to a higher rate of a favourable functional outcome (OR 1.44; 95\% CI 1.10 to $1.89 ; \mathrm{p}=0.001$ ), despite a higher risk of symptomatic haemorrhagic transformation with statin use (OR 1.63; 95\% CI 1.04 to 2.56; $\mathrm{p}=0.035) .{ }^{92}$ The protective effect of statins towards a better functional outcome and against stroke recurrence may lie in their pleiotropic effects, for instance, reducing the concentration of low-density lipoprotein cholesterol, mild effect in lowering the blood pressure and anti-inflammatory effects. ${ }^{91}$ Moreover, it has been indicated in small studies that prestroke use of statins might be independently associated with better collateral circulation in cardioembolic, large artery atherosclerotic strokes or strokes of unknown aetiologies, possibly through increasing nitric oxide synthesis and promoting ischaemia-induced neovascularisation. ${ }^{93} 94$ The pleiotropic effects of statins in patients with stroke, including that in boosting collateral flow, warrant further investigation.

\section{Urinary kallidinogenase and dl-3-n-butylphthalide}

Urinary kallidinogenase increases cerebral blood flow velocity and reduces the infarct size in an experimental stroke model by thread occlusion of MCA in rats. ${ }^{95} \mathrm{~A}$ small, open-label, controlled, prospective study implied that short-term application of human urinary kallidinogenase could upregulate vascular endothelial growth factor and apelin expression, shorten the MTT in MR perfusion, and improve the 3-month functional outcome, as compared with control, among patients with acute stroke.$^{96}$ According to a systematic review and meta-analysis of 24 trials with 2433 patients published in 2012, human urinary kallidinogenase injection reduced death or dependency in two trials (RR $0.69 ; 95 \%$ CI 0.55 to 0.86 ) and increased the rate of neurological improvement after treatment based on data from 20 trials (2117 patients) (RR 1.56; 95\% CI 1.44 to 1.70 ) as compared with control, while the risks of non-fatal intracranial haemorrhage or death were not significantly different between those treated with human urinary kallidinogenase or controls. ${ }^{97}$

Administration of dl-3n-butylphthalidehas has been indicated to increase the number of perfused microvessels, enhance cerebral blood flow, and reduce the incidence and infarct size in rat stroke models. ${ }^{98}{ }^{99}$ A systematic review and meta-analysis published in 2010 reported more significant neurological improvement in patients treated with dl-3n-butylphthalide than controls (21 studies of 2123 patients), while there was no report on the rates of death of dependency in these studies. ${ }^{100}$ A recent RCT of 170 patients reported that dl-3n-butylphthalide plus standard medical treatment had a mild effect in enhancing neurological recovery in patients with acute ischaemic stroke as compared with standard medical treatment alone, which 
was correlated with a significantly higher level of circulating endothelial progenitor cells that may promote angiogenesis and neovascularisation in those treated with dl-3n-butylphthalide. ${ }^{101}$ Another RCT compared the efficacies of 14-day infusion of dl-3n-butylphthalide followed by a dl-3n-butylphthalide capsule, 14-day infusion of dl-3n-butylphthalide followed by aspirin, or a 14-day infusion of ozagrel followed by aspirin, among 573 patients with acute ischaemic stroke treated starting within 48 hours of onset. The study found a significant better functional outcome at 90 days in patients treated with dl-3n-butylphthalide for 90 days than those treated with ozagrel $(\mathrm{p}<0.001) .{ }^{102}$ However, there are doubts in the study findings, since none of the treatment assignments in this study were standard medical treatment in clinical practice.

Overall, although urinary kallidinogenase and dl-3n-butylphthalide have shown promising effects in promoting collateral circulation, increasing cerebral blood flow and improving the functional outcome after ischaemic stroke in animal models and in preliminary clinical studies, flawed study design of previous relevant studies urges further investigation on the effects and pharmaceutical mechanisms of the two novel medications in patients with stroke.

\section{Drug-induced hypertension}

Results of animal studies hinted that drug-induced mild hypertension could increase cerebral blood flow and cerebral oxygen metabolism in the infarct core and penumbra, which might lead to a smaller infarct size. ${ }^{103}$ By far, data are limited regarding the safety and efficacy of induced hypertension therapy in patients with ischaemic stroke. A couple of small studies showed a possible favourable effect of induced hypertension (using phenylephrine to increase the systolic blood pressure to a target of $160-200 \mathrm{~mm} \mathrm{Hg}$ or increase the mean blood pressure by $10 \%-20 \%$ ) over early neurological improvement in patients with acute ischaemic stroke with large artery occlusion or significant perfusion-diffusion mismatch. ${ }^{104}$ Findings of a currently ongoing multicentre, randomised, open-label trial, the Therapeutic INduced HYPERTENSION in acute non-cardioembolic ischaemic stroke (SETIN-HYPERTENSION; ClinicalTrials.gov identifier: NCT01600235) trial, may yield valuable data for this topic.

\section{Hypervolaemic treatment}

Preclinical studies and pilot clinical studies showed possible neuroprotective effect of hypervolaemic treatment using albumin in acute ischaemic stroke, which improved cerebral perfusion in regions with critically reduced cerebral blood flow that might lead to a better functional outcome. ${ }^{105}$ However, early initiation of albumin treatment has been shown to have no additional clinical benefit versus isotonic saline in adult patients with ischaemic stroke with a baseline NIHSS score of 6 or higher who were treated within 5 hours of symptoms onset, in a multicentre, double-blinded RCT, the Albumin in Acute Ischemic Stroke Trial (ALIAS) trial. The ALIAS trial was stopped early at 841 patients since no difference was identified in the albumin and saline treatment groups in the primary outcome (mRS $0-1$ or NIHSS scores $0-1$ at 90 days; $44 \%$ vs $44 \%$; RR 0.96 ; $95 \%$ CI 0.84 to $1.10)$, while there were more events of pulmonary oedema or congestive heart failure within 48 hours (13\% vs $1 \%$; RR $10.8 ; 95 \%$ CI 4.37 to 26.72 ) and symptomatic intracranial haemorrhage within 24 hours ( $4 \%$ vs $2 \%$; RR 2.42; $95 \%$ CI 1.02 to 5.78 ) in those treated with albumin than isotonic saline. ${ }^{106}$

\section{Recommendations}

1. Direct EC-IC bypass surgery is not recommended for patients with general ischaemic stroke or TIA with symptomatic intracranial atherosclerotic stenosis or occlusion (class III; level of evidence A). Further investigation is needed on the safety and efficacy of direct EC-IC bypass surgery in carefully selected patients with large artery atherosclerotic stroke with significantly compromised cerebral blood flow and/or cerebrovascular reactivity (class IIb; level of evidence $\mathrm{C}$ ). The safety and efficacy of EDAS in patients with symptomatic intracranial atherosclerotic stenosis or occlusion warrant further verification (class IIb; level of evidence C).

2. Although the NeuroFlo treatment shows additional benefit over medical treatment in certain subgroups of patients with stroke, it is not recommended in patients with general stroke based on current evidence (class III; level of evidence A).

3. ECP is safe and possibly effective to augment cerebral blood flow in patients with acute ischaemic stroke, while the clinical benefit needs further investigation (class IIb; level of evidence $\mathrm{C}$ ).

4. Lying-flat head positioning may increase cerebral blood flow as compared with upright head positioning, but no conclusion could be drawn based on current evidence regarding the effects of different head positions on clinical outcomes of patients with ischaemic stroke (class IIb; level of evidence $\mathrm{C}$ ).

5. RIPC is safe and feasible, and may benefit patients with stroke with symptomatic ICAS. Further investigation is under way (class IIb; level of evidence $\mathrm{C}$ ).

6. Statin treatment may enhance collateral flow and have other protective effects in patients with non-cardioembolic or cardioembolic stroke. It is reasonable to use statins in patients with non-cardioembolic stroke (class IIa; level of evidence B), and possibly reasonable to use in patients with cardioembolic stroke as well (class IIb; level of evidence $\mathrm{C}$ ).

7. Urinary kallidinogenase and dl-3n-butylphthalide have shown promising effects in improving cerebral blood flow and the functional outcome after ischaemic stroke, but further investigation is needed (class IIa; level of evidence B).

8. The safety and efficacy of induced hypertensive therapy in patients with acute ischaemic stroke with large artery occlusion and hypoperfusion are not clear based on current evidence (class IIb; level of evidence C). 
9. Hypervolaemic treatment is not recommended for patients with general acute ischaemic stroke based on current evidence (class III; level of evidence A).

\section{PERSPECTIVES}

With rapid progress in neuroimaging and computational techniques in recent years, various novel and non-invasive methods are emerging to evaluate cerebral collateral circulation, haemodynamics, metabolism and neuronal functions. Advances in the assessment and interventions for cerebral collateral circulation will facilitate more precise diagnosis and risk stratification, and more patient-specific management of patients with stroke, which will lead to better prognosis of affected patients. Cerebral collateral circulation is becoming a hot spot in stroke research. The following directions are promising in the near future.

1. Advancing multimodal imaging and postprocessing technologies and optimising methods and criteria, for more accurate transient and dynamic evaluation of cerebral collateral circulation and haemodynamics; verifying the role of collateral circulation in governing the functional outcomes and recurrent risks in patients with ischaemic stroke with and without large artery atherosclerotic disease.

There are ongoing research projects targeting at these topics. For instance, the cerebral collateral circulation evaluation and prediction for acute cerebral ischaemia (COLLATERAL) study, a prospective, multicentre, nested case-control study, plans to assess the collateral status and its prognostic value in 3750 patients with acute ischaemic stroke, in which study multiphase CTA is used for collateral assessment. In addition, studies are under way to compare the values of novel imaging and computational methods versus conventional methods in gauging collateral circulation and cerebral haemodynamics in patients with stroke with symptomatic intracranial atherosclerotic disease, for instance, pseudocontinuous ASL MR perfusion imaging to assess cerebral perfusion and collateral circulation, and angiographic imaging-based computational fluid dynamics modelling to quantify cerebral haemodynamic metrics such as intraluminal pressure, flow velocities, wall shear and others. These studies will provide insights for simple, non-invasive but more accurate assessment of collateral circulation and may yield higher prognostic values.

2. Verifying the role of collateral circulation in governing response to acute endovascular therapy in patients with stroke towards more reasonable selection of patients for endovascular treatment in an extended time window.

Evidence is rapidly accumulating regarding the remarkable benefit of acute endovascular treatment for patients with stroke, which urges an extension in the time window for treatment. When the DAWN trial and further studies ultimately extend the time window to 24 hours or beyond, selecting suitable patients to treat will be essential in maximising the benefit of endovascular treatment, while the pretreatment collateral status could be a key factor to be taken into account. Currently, multicentre, prospective studies are under way to further verify the role of collateral circulation in altering the imaging and clinical outcomes after endovascular treatment, and/or to explore the role of baseline collateral status in influencing clinical decisions for endovascular treatment, such as the Stroke: An Evaluation of Thrombectomy in the Ageing Brain (STABILISE), MR-based Collateral Imaging to Predict Response to Endovascular Treatment of Stroke (FAST-COLL), Measuring Collaterals With Multiphase CT Angiography in Patients With Ischemic Stroke (PRove-IT), and Optimising Patient's Selection for Endovascular Treatment in Acute Ischemic Stroke (SELECT) trials. CT-based and/or MR-based methods for collateral assessment are tested in these trials. Findings of these trials will provide clues for a better strategy in clinical decisions to pursue endovascular treatment in ischaemic stroke, especially in an extended time window.

3. Testing currently existing and promising methods, and in the meantime exploring new methods, to improve collateral circulation.

Although various pharmaceutical and non-pharmaceutical interventions have shown promising effects in augmenting cerebral collateral circulation and cerebral blood flow in patients with stroke, current evidence is limited to support the application of these interventions in clinical practice. Further investigation is in progress on the effects of EDAS, ECP, RIPC, and medications such as statins, urinary kallidinogenase and dl-3n-butylphthalide in improving collateral circulation and prognosis of certain subgroups of patients with stroke. Efforts are also needed to pursue novel methods in this area.

4. Establishing interdisciplinary imaging processing and assessment platforms and promoting telestroke systems, for timely and accurate patient assessment and triage in comprehensive stroke centres, as well as in lower level stroke centres.

With prompt advances of imaging and artificial intelligence technologies in recent years and the years to come, bioengineers and computer scientists are increasingly involved in medical imaging processing and assessment. Therefore, establishment of interdisciplinary imaging platforms is ultimately inevitable for timely and accurate imaging processing and evaluation in acute ischaemic stroke. On the other hand, application of telestroke systems will facilitate accurate diagnosis and triage of patients with stroke presenting at lower level stroke centres, so that such patients could be treated properly on site or be transferred to a higher level stroke centre for more advanced treatments (such as acute endovascular treatment) that are not available on site. In addition, incorporating resources from multiple disciplines and centres will provide big 
data for research into precise stratification and management of relevant patients in the near future.

Correction notice This article has been corrected since it published Online First. The author affiliations have been corrected.

Acknowledgements We thank the panel experts for their comments on the consensus.

Collaborators Jian-Ming Cai (Department of Radiology, The General Hospital of People's Liberation Army), Tao Chen (Department of Neurology, The First Affiliated Hospital of Kunming Medical University), Xin Chen (Department of Neurology, Huashan Hospital, Fudan University, Shanghai), Jing Ding (Department of Neurology, Zhongshan Hospital, Fudan University, Shanghai), Lian-Bo Gao (Department of Neurology, The Fourth Affiliated Hospital of China Medical University), Yang-Tai Guan (Department of Neurology, Renji Hospital, Shanghai Jiao Tong University School of Medicine), Lawrence KS Wong (Department of Medicine \& Therapeutics, The Chinese University of Hong Kong, Prince of Wales Hospital, Shatin, Hong Kong), Li-An Huang (Department of Neurology, The First Affiliated Hospital, Jinan University), Xin-Yi Leng (Department of Medicine \& Therapeutics, The Chinese University of Hong Kong, Prince of Wales Hospital, Shatin, Hong Kong), Guo-Zhong Li (Department of Neurology, The First Affiliated Hospital of Harbin Medical University), Jing-Wei Li (Department of Neurology, Nanjing Drum Tower Hospital, Nanjing University Medical School), Ye Li (Paul C Lauterbur Research Center for Biomedical Imaging, Shenzhen Institute of Advanced Technology, Chinese Academy of Science), Jia Liu (Shenzhen Institute of Advanced Technology, Chinese Academy of Science), Jun-Yan Liu (Department of Neurology, The Third Affiliated Hospital, Hebei Medical University), Li-Ping Liu (Department of Neurology, Beijing Tiantan Hospital, Capital Medical University), Jie Lu (Department of Radiology, Xuanwu Hospital, Capital Medical University), Qi-Zheng Lu (The Third Affiliated Hospital, Sun Yat-sen University), Yue-Song Pan (Department of Neurology, Beijing Tiantan Hospital, Capital Medical University), Yue-Hua Pu (Department of Neurology, Beijing Tiantan Hospital, Capital Medical University), Xiao-Yi Wang (Shenzhen Delica Medical Equipment), Xin Wang (Department of Neurology, Zhongshan Hospital, Fudan University, Shanghai), Yi-Long Wang (Department of Neurology, Beijing Tiantan Hospital, Capital Medical University), An-Ding Xu (Department of Neurology, The First Affiliated Hospital, Jinan University), Guo-Yuan Yang (Shanghai Med-X Engineering Center for Medical Equipment and Technology), Su-Ming Zhang (Tongji Hospital, Tongji Medical College, Huazhong University of Science and Technology) and Xi-Hai Zhao (Center for Biomedical Imaging Research, Tsinghua University).

Contributors LL, JD, XL, YP, L-AH, AX, KSLW: wrote the manuscript. XW, YW: critical revision of the manuscript.

Funding The authors have not declared a specific grant for this research from any funding agency in the public, commercial or not-for-profit sectors.

Competing interests None declared.

Patient consent Not required.

Provenance and peer review Commissioned; externally peer reviewed.

Open access This is an open access article distributed in accordance with the Creative Commons Attribution Non Commercial (CC BY-NC 4.0) license, which permits others to distribute, remix, adapt, build upon this work non-commercially, and license their derivative works on different terms, provided the original work is properly cited and the use is non-commercial. See: http://creativecommons.org/ licenses/by-nc/4.0/

(c) Article author(s) (or their employer(s) unless otherwise stated in the text of the article) 2018. All rights reserved. No commercial use is permitted unless otherwise expressly granted.

\section{REFERENCES}

1. Bang OY, Saver JL, Kim SJ, et al. Collateral flow predicts response to endovascular therapy for acute ischemic stroke. Stroke 2011:42:693-9.

2. Goyal M, Demchuk AM, Menon BK, et al. Randomized assessment of rapid endovascular treatment of ischemic stroke. $N$ Engl J Med 2015;372:1019-30.

3. Bang OY, Saver JL, Kim SJ, et al. Collateral flow averts hemorrhagic transformation after endovascular therapy for acute ischemic stroke. Stroke 2011;42:2235-9.

4. Liebeskind DS, Cotsonis GA, Saver JL, et al. Collaterals dramatically alter stroke risk in intracranial atherosclerosis. Ann Neurol 2011;69:963-74.
5. Fanou EM, Knight J, Aviv RI, et al. Effect of Collaterals on Clinical Presentation, Baseline Imaging, Complications, and Outcome in Acute Stroke. AJNR Am J Neuroradiol 2015;36:2285-91.

6. Liu LP, Xu AD, Wong LK, et al. Chinese consensus statement on the evaluation and intervention of collateral circulation for ischemic stroke. CNS Neurosci Ther 2014;20:202-8.

7. Liebeskind DS. Collateral circulation. Stroke 2003;34:2279-84.

8. Liebeskind DS. Imaging the collaterome: a stroke renaissance. Curr Opin Neurol 2015;28:1-3.

9. McVerry F, Liebeskind DS, Muir KW. Systematic review of methods for assessing leptomeningeal collateral flow. AJNR Am J Neuroradiol 2012;33:576-82.

10. Martinon E, Lefevre $\mathrm{PH}$, Thouant $\mathrm{P}$, et al. Collateral circulation in acute stroke: assessing methods and impact: a literature review. $J$ Neuroradiol 2014;41:97-107.

11. Alexandrov AV, Sloan MA, Tegeler $\mathrm{CH}$, et al. Practice standards for transcranial Doppler (TCD) ultrasound. Part II. Clinical indications and expected outcomes. J Neuroimaging 2012;22:215-24.

12. Brunser AM, Mansilla E, Hoppe A, et al. The Role of TCD in the Evaluation of Acute Stroke. J Neuroimaging 2016;26:420-5.

13. Müller $M$, Hermes $M$, Brückmann $H$, et al. Transcranial Doppler ultrasound in the evaluation of collateral blood flow in patients with internal carotid artery occlusion: correlation with cerebral angiography. AJNR Am J Neuroradiol 1995;16:195-202.

14. Kim Y, Sin DS, Park HY, et al. Relationship between flow diversion on transcranial Doppler sonography and leptomeningeal collateral circulation in patients with middle cerebral artery occlusive disorder. $J$ Neuroimaging 2009;19:23-6.

15. Hendrikse J, Klijn CJ, van Huffelen AC, et al. Diagnosing cerebral collateral flow patterns: accuracy of non-invasive testing. Cerebrovasc Dis 2008;25:430-7.

16. Han A, Yoon DY, Chang SK, et al. Accuracy of CT angiography in the assessment of the circle of Willis: comparison of volumerendered images and digital subtraction angiography. Acta Radiol 2011;52:889-93.

17. Smit EJ, Vonken EJ, van der Schaaf IC, et al. Timing-invariant reconstruction for deriving high-quality CT angiographic data from cerebral CT perfusion data. Radiology 2012;263:216-25.

18. Smit EJ, Vonken EJ, van Seeters T, et al. Timing-invariant imaging of collateral vessels in acute ischemic stroke. Stroke 2013;44:2194-9.

19. Kaschka IN, Kloska SP, Struffert T, et al. Clot Burden and Collaterals in Anterior Circulation Stroke: Differences Between Single-Phase CTA and Multi-phase 4D-CTA. Clin Neuroradiol 2016;26:309-15.

20. Beyer SE, Thierfelder KM, von Baumgarten L, et al. Strategies of collateral blood flow assessment in ischemic stroke: prediction of the follow-up infarct volume in conventional and dynamic CTA. AJNR Am J Neuroradiol 2015;36:488-94.

21. van den Wijngaard IR, Holswilder G, Wermer MJ, et al. Assessment of Collateral Status by Dynamic CT Angiography in Acute MCA Stroke: Timing of Acquisition and Relationship with Final Infarct Volume. AJNR Am J Neuroradiol 2016;37:1231-6.

22. Menon BK, d'Esterre CD, Qazi EM, et al. Multiphase CT Angiography: A New Tool for the Imaging Triage of Patients with Acute Ischemic Stroke. Radiology 2015;275:510-20.

23. Rusanen H, Saarinen JT, Sillanpää N. Collateral Circulation Predicts the Size of the Infarct Core and the Proportion of Salvageable Penumbra in Hyperacute Ischemic Stroke Patients Treated with Intravenous Thrombolysis. Cerebrovasc Dis 2015;40:182-90.

24. Vagal A, Menon BK, Foster LD, et al. Association Between CT Angiogram Collaterals and CT Perfusion in the Interventional Management of Stroke III Trial. Stroke 2016;47:535-8.

25. Campbell BC, Mitchell PJ, Kleinig TJ, et al. Endovascular therapy for ischemic stroke with perfusion-imaging selection. N Engl J Med 2015;372:1009-18.

26. Lansberg MG, Christensen S, Kemp S, et al. Computed tomographic perfusion to Predict Response to Recanalization in ischemic stroke. Ann Neurol 2017;81:849-56.

27. Albers GW, Marks MP, Kemp S, Marks SK, et al. Thrombectomy for Stroke at 6 to 16 Hours with Selection by Perfusion Imaging. N Engl $J$ Med 2018;378:708-18.

28. Higashida RT, Furlan AJ, Roberts $\mathrm{H}$, et al. Trial design and reporting standards for intra-arterial cerebral thrombolysis for acute ischemic stroke. Stroke 2003;34:e109-e137.

29. Singer OC, Berkefeld J, Nolte $\mathrm{CH}$, et al. Collateral vessels in proximal middle cerebral artery occlusion: the ENDOSTROKE study. Radiology 2015;274:851-8.

30. Singer OC, Berkefeld J, Nolte $\mathrm{CH}$, et al. Mechanical recanalization in basilar artery occlusion: the ENDOSTROKE study. Ann Neurol 2015;77:415-24. 
31. Liebeskind DS, Tomsick TA, Foster LD, et al. Collaterals at angiography and outcomes in the Interventional Management of Stroke (IMS) III trial. Stroke 2014;45:759-64.

32. Liebeskind DS, Jahan R, Nogueira RG, et al. Impact of collaterals on successful revascularization in Solitaire FR with the intention for thrombectomy. Stroke 2014;45:2036-40.

33. Christoforidis GA, Mohammad Y, Kehagias D, et al. Angiographic assessment of pial collaterals as a prognostic indicator following intra-arterial thrombolysis for acute ischemic stroke. AJNR Am J Neuroradiol 2005;26:1789-97.

34. Christoforidis GA, Karakasis C, Mohammad Y, et al. Predictors of hemorrhage following intra-arterial thrombolysis for acute ischemic stroke: the role of pial collateral formation. AJNR Am J Neuroradiol 2009;30:165-70.

35. Miteff $F$, Levi CR, Bateman GA, et al. The independent predictive utility of computed tomography angiographic collateral status in acute ischaemic stroke. Brain 2009;132:2231-8.

36. Maas MB, Lev MH, Ay H, et al. Collateral vessels on CT angiography predict outcome in acute ischemic stroke. Stroke 2009:40:3001-5.

37. Tan IY, Demchuk AM, Hopyan J, et al. CT angiography clot burden score and collateral score: correlation with clinical and radiologic outcomes in acute middle cerebral artery infarct. AJNR Am J Neuroradiol 2009;30:525-31.

38. Menon BK, Smith EE, Modi J, et al. Regional leptomeningeal score on CT angiography predicts clinical and imaging outcomes in patients with acute anterior circulation occlusions. AJNR Am J Neuroradiol 2011;32:1640-5.

39. Menon BK, O'Brien B, Bivard A, et al. Assessment of leptomeningeal collaterals using dynamic $\mathrm{CT}$ angiography in patients with acute ischemic stroke. J Cereb Blood Flow Metab 2013;33:365-71.

40. Menon BK, Qazi E, Nambiar V, et al. Differential Effect of Baseline Computed Tomographic Angiography Collaterals on Clinical Outcome in Patients Enrolled in the Interventional Management of Stroke III Trial. Stroke 2015;46:1239-44.

41. Yeo LL, Paliwal P, Teoh HL, et al. Assessment of intracranial collaterals on CT angiography in anterior circulation acute ischemic stroke. AJNR Am J Neuroradiol 2015;36:289-94.

42. Seker F, Potreck A, Möhlenbruch M, et al. Comparison of four different collateral scores in acute ischemic stroke by CT angiography. J Neurointerv Surg 2016;8:1116-8.

43. Jauch EC, Saver JL, Adams HP, et al. Guidelines for the early management of patients with acute ischemic stroke: a guideline for healthcare professionals from the American Heart Association/ American Stroke Association. Stroke 2013;44:870-947.

44. Powers WJ, Derdeyn CP, Biller J, et al. American Heart Association/ American Stroke Association focused update of the 2013 Guidelines for the early management of patients with acute ischemic stroke regarding endovascular treatment: a guideline for healthcare professionals from the American Heart Association/ American Stroke Association. Stroke 2015;2015:3020-35.

45. Chinese Society of Neurology of Chinese Medical Association, Cerebrovascular Disease Study Group, Chinese Society of Neurology, Chinese Medical Association. The 2014 Chinese guidelines for diagnosis and management of patients with acute ischemic stroke. Chin J Neurol 2015;48:246-57.

46. Chinese Stroke Association, Chinese Interventional Neuroradiology Society of Chinese Stroke Association, Intervention Study Group of Stroke Prevention and Control Society of Chinese Preventive Medicine Association. The 2015 Chinese Guidelines for endovascular treatment of patients with acute ischemic stroke. Chin J Stroke 2015;10:590-606.

47. Kim YS, Meyer JS, Garami Z, et al. Flow diversion in transcranial Doppler ultrasound is associated with better improvement in patients with acute middle cerebral artery occlusion. Cerebrovasc Dis 2006;21:74-8.

48. Campbell BC, Christensen S, Tress BM, et al. Failure of collateral blood flow is associated with infarct growth in ischemic stroke. $J$ Cereb Blood Flow Metab 2013;33:1168-72.

49. Leng X, Lan L, Liu L, et al. Good collateral circulation predicts favorable outcomes in intravenous thrombolysis: a systematic review and meta-analysis. Eur J Neurol 2016:23:1738-49.

50. Jovin TG, Chamorro A, Cobo E, et al. Thrombectomy within 8 hours after symptom onset in ischemic stroke. $N$ Engl $\mathrm{J} \mathrm{Med}$ 2015;372:2296-306

51. Saver JL, Goyal M, Bonafe A, et al. Stent-retriever thrombectomy after intravenous t-PA vs. t-PA alone in stroke. N Engl J Med 2015;372:2285-95

52. Jovin TG, Nogueira RG. for the DAWN investigators. DAWN in full daylight (DWI or CTP Assessment with Clinical Mismatch in the Triage of Wake Up and Late Presenting Strokes Undergoing Neurointervention). Eur Stroke J 2017;2:494.

53. Jovin TG, Saver JL, Ribo M, et al. Diffusion-weighted imaging or computerized tomography perfusion assessment with clinical mismatch in the triage of wake up and late presenting strokes undergoing neurointervention with Trevo (DAWN) trial methods. Int $J$ Stroke 2017:12:641-52.

54. Leng X, Fang H, Leung TW, et al. Impact of collaterals on the efficacy and safety of endovascular treatment in acute ischaemic stroke: a systematic review and meta-analysis. J Neurol Neurosurg Psychiatry 2016;87:537-44.

55. Leng X, Fang $\mathrm{H}$, Leung TW, et al. Impact of Collateral Status on Successful Revascularization in Endovascular Treatment A Systematic Review and Meta-Analysis. Cerebrovasc Dis 2016;41:27-34

56. Liebeskind DS. Collateral lessons from recent acute ischemic stroke trials. Neurol Res 2014;36:397-402.

57. Liggins JT, Mlynash M, Jovin TG, et al. Interhospital variation in reperfusion rates following endovascular treatment for acute ischemic stroke. J Neurointerv Surg 2015;7:231-3.

58. Wong KS, Huang YN, Gao S, et al. Intracranial stenosis in Chinese patients with acute stroke. Neurology 1998;50:812-3.

59. Wong LK. Global burden of intracranial atherosclerosis. Int J Stroke 2006;1:158-9

60. Wang Y, Zhao X, Liu L, et al. Prevalence and outcomes of symptomatic intracranial large artery stenoses and occlusions in China: the Chinese Intracranial Atherosclerosis (CICAS) Study. Stroke 2014:45:663-9.

61. Wang $Y$, Wang $Y$, Zhao $X$, et al. Clopidogrel with aspirin in acute minor stroke or transient ischemic attack. N Engl $\mathrm{J} \mathrm{Med}$ 2013;369:11-19.

62. Liu L, Wong KS, Leng $X$, et al. Dual antiplatelet therapy in stroke and ICAS: Subgroup analysis of CHANCE. Neurology 2015;85:1154-62.

63. Liebeskind D, Cotsonis G, Saver J, et al. Warfarin-Aspirin Symptomatic Intracranial Disease (WASID) Investigators. Collaterals dramatically alter stroke risk in intracranial atherosclerosis. Ann Neurol 2011;69:963-74.

64. Lau AY, Wong EH, Wong A, et al. Significance of good collateral compensation in symptomatic intracranial atherosclerosis. Cerebrovasc Dis 2012;33:517-24.

65. Liu D, Li Y, Shi Z, et al. Presence of anterior temporal artery associates with good outcome in acute atherosclerotic M1-middle cerebral artery occlusion. Neuroradiology 2014;56:1023-30.

66. Song J, Ma Z, Meng H, et al. Distal hyperintense vessels alleviate insula infarction in proximal middle cerebral artery occlusion. Int $J$ Neurosci 2016:126:1030-5.

67. Kim JM, Jung KH, Sohn CH, et al. Intracranial plaque enhancement from high resolution vessel wall magnetic resonance imaging predicts stroke recurrence. Int J Stroke 2016;11:171-9.

68. Lee WJ, Jung KH, Ryu YJ, et al. Utility of digital subtraction angiography-based collateral evaluation in medically treated acute symptomatic basilar artery stenosis. Eur $J$ Neurol 2017;24:1148-55.

69. Kim KM, Kang HS, Lee WJ, et al. Clinical significance of the circle of Willis in intracranial atherosclerotic stenosis. $J$ Neurointerv Surg 2016;8:251-5.

70. Low SW, Teo K, Lwin S, et al. Improvement in cerebral hemodynamic parameters and outcomes after superficial temporal artery-middle cerebral artery bypass in patients with severe stenoocclusive disease of the intracranial internal carotid or middle cerebral arteries. J Neurosurg 2015;123:662-9.

71. EC/IC Bypass Study Group. Failure of extracranial-intracrania arterial bypass to reduce the risk of ischemic stroke. Results of an international randomized trial. N Engl J Med 1985;313:1191-200.

72. Powers WJ, Clarke WR, Grubb RL, et al. Extracranial-intracranial bypass surgery for stroke prevention in hemodynamic cerebral ischemia: the Carotid Occlusion Surgery Study randomized trial. JAMA 2011;306:1983-92.

73. JET Study Group. Japanese EC-IC Bypass Trial (JET Study): The second interim analysis. Surg Cereb Stroke 2002;30:434-7.

74. Kernan WN, Ovbiagele B, Black HR, et al. Guidelines for the prevention of stroke in patients with stroke and transient ischemic attack: a guideline for healthcare professionals from the American Heart Association/American Stroke Association. Stroke 2014:45:2160-236.

75. Dusick JR, Liebeskind DS, Saver JL, et al. Indirect revascularization for nonmoyamoya intracranial arterial stenoses: clinical and angiographic outcomes. J Neurosurg 2012;117:94-102.

76. Gonzalez NR, Dusick JR, Connolly M, et al. Encephaloduroarteriosynangiosis for adult intracranial arterial 
steno-occlusive disease: long-term single-center experience with 107 operations. J Neurosurg 2015;123:654-61.

77. Shuaib A, Bornstein NM, Diener HC, et al. Partial aortic occlusion for cerebral perfusion augmentation: safety and efficacy of NeuroFlo in Acute Ischemic Stroke trial. Stroke 2011;42:1680-90.

78. Shuaib A, Schwab S, Rutledge JN, et al. Importance of proper patient selection and endpoint selection in evaluation of new therapies in acute stroke: further analysis of the SENTIS trial. J Neurointerv Surg 2013;5 Suppl 1:i21-i24.

79. Alexandrov AW, Ribo M, Wong KS, et al. Perfusion augmentation in acute stroke using mechanical counter-pulsation-phase lla: effect of external counterpulsation on middle cerebral artery mean flow velocity in five healthy subjects. Stroke 2008;39:2760-4.

80. Han JH, Leung TW, Lam WW, et al. Preliminary findings of external counterpulsation for ischemic stroke patient with large artery occlusive disease. Stroke 2008;39:1340-3.

81. Guluma KZ, Liebeskind DS, Raman R, et al. Feasibility and safety of using external counterpulsation (ECP) to augment cerebral blood flow in acute ischemic stroke - the CUFFS trial. J Stroke Cerebrovasc Dis 2015;24:2596-604.

82. Lin W, Xiong L, Han J, et al. External counterpulsation augments blood pressure and cerebral flow velocities in ischemic stroke patients with cerebral intracranial large artery occlusive disease. Stroke 2012;43:3007-11.

83. Hunter AJ, Snodgrass SJ, Quain D, et al. HOBOE (Head-of-Bed Optimization of Elevation) Study: association of higher angle with reduced cerebral blood flow velocity in acute ischemic stroke. Phys Ther 2011;91:1503-12.

84. Olavarría VV, Arima $\mathrm{H}$, Anderson CS, et al. Head position and cerebral blood flow velocity in acute ischemic stroke: a systematic review and meta-analysis. Cerebrovasc Dis 2014;37:401-8.

85. Billot L, Woodward M, Arima $\mathrm{H}$, et al. Statistical analysis plan for the Head Position in Stroke Trial (HeadPoST): An international cluster cross-over randomized trial. Int J Stroke 2017:12:667-70.

86. Anderson CS, Arima H, Lavados P, et al. Cluster-Randomized, Crossover Trial of Head Positioning in Acute Stroke. N Engl J Med 2017;376:2437-47.

87. Meng R, Asmaro K, Meng L, et al. Upper limb ischemic preconditioning prevents recurrent stroke in intracranial arterial stenosis. Neurology 2012;79:1853-61.

88. Meng R, Ding Y, Asmaro K, et al. Ischemic Conditioning Is Safe and Effective for Octo- and Nonagenarians in Stroke Prevention and Treatment. Neurotherapeutics 2015;12:667-77.

89. Terpolilli NA, Kim SW, Thal SC, et al. Inhalation of nitric oxide prevents ischemic brain damage in experimental stroke by selective dilatation of collateral arterioles. Circ Res 2012;110:727-38.

90. Henninger N, Fisher M. Stimulating circle of Willis nerve fibers preserves the diffusion-perfusion mismatch in experimental stroke. Stroke 2007;38:2779-86.
91. Amarenco P, Lavallée P, Touboul PJ, et al. Stroke prevention, blood cholesterol, and statins. Lancet Neurol 2004;3:271-8.

92. Hong KS, Lee JS. Statins in acute ischemic stroke: a systematic review. J Stroke 2015;17:282-301.

93. Ovbiagele B, Saver JL, Starkman S, et al. Statin enhancement of collateralization in acute stroke. Neurology 2007;68:2129-31.

94. Lee MJ, Bang OY, Kim SJ, et al. Role of statin in atrial fibrillationrelated stroke: an angiographic study for collateral flow. Cerebrovasc Dis 2014;37:77-84.

95. Li C, Min Z, Zhan Y, et al. Use of laser speckle imaging to study effects of urinary kallidinogenase on cerebral blood flow following cerebral infarction in rats. Chin J Neurol 2010;43:732-6.

96. Li J, Chen Y, Zhang X, et al. Human urinary kallidinogenase improves outcome of stroke patients by shortening mean transit time of perfusion magnetic resonance imaging. J Stroke Cerebrovasc Dis 2015;24:1730-7.

97. Zhang C, Tao W, Liu M, et al. Efficacy and safety of human urinary kallidinogenase injection for acute ischemic stroke: a systematic review. J Evid Based Med 2012;5:31-9.

98. Zhang Y, Wang L, Li J, et al. 2-(1-Hydroxypentyl)-benzoate increases cerebral blood flow and reduces infarct volume in rats model of transient focal cerebral ischemia. J Pharmacol Exp Ther 2006;317:973-9.

99. Liu CL, Liao SJ, Zeng JS, et al. dl-3n-butylphthalide prevents stroke via improvement of cerebral microvessels in RHRSP. J Neurol Sci 2007;260:106-13.

100. Wang D, Liu M, Wu B, et al. DI-3-butylphthalide for acute ischemic stroke: a systematic review. Chin J Evid Based Med 2010:10:189-95.

101. Zhao H, Yun W, Zhang Q, et al. Mobilization of circulating endothelial progenitor cells by dl-3-n-butylphthalide in acute ischemic stroke patients. J Stroke Cerebrovasc Dis 2016;25:752-60.

102. Cui LY, Zhu YC, Gao S, et al. Ninety-day administration of dl-3-nbutylphthalide for acute ischemic stroke: a randomized, doubleblind trial. Chin Med J 2013;126:3405-10.

103. Shin HK, Nishimura M, Jones PB, et al. Mild induced hypertension improves blood flow and oxygen metabolism in transient focal cerebral ischemia. Stroke 2008;39:1548-55.

104. McManus M, Liebeskind DS. Blood pressure in acute ischemic stroke. J Clin Neurol 2016;12:137-46.

105. Palesch YY, Hill MD, Ryckborst KJ, et al. The ALIAS Pilot Trial: a dose-escalation and safety study of albumin therapy for acute ischemic stroke--II: neurologic outcome and efficacy analysis. Stroke 2006;37:2107-14.

106. Ginsberg MD, Palesch YY, Hill MD, et al. High-dose albumin treatment for acute ischaemic stroke (ALIAS) Part 2: a randomised, double-blind, phase 3, placebo-controlled trial. Lancet Neurol 2013;12:1049-58. 